\title{
An enhanced visualization of DBT imaging using blind deconvolution and total variation minimization regularization
}

\author{
Ana M. Mota*, Matthew J. Clarkson, Pedro Almeida and Nuno Matela
}

\begin{abstract}
Digital Breast Tomosynthesis (DBT) presents out-of-plane artifacts caused by features of high intensity. Given observed data and knowledge about the point spread function (PSF), deconvolution techniques recover data from a blurred version. However, a correct PSF is difficult to achieve and these methods amplify noise. When no information is available about the PSF, blind deconvolution can be used. Additionally, Total Variation (TV) minimization algorithms have achieved great success due to its virtue of preserving edges while reducing image noise. This work presents a novel approach in DBT through the study of outof-plane artifacts using blind deconvolution and noise regularization based on TV minimization. Gradient information was also included. The methodology was tested using real phantom data and one clinical data set. The results were investigated using conventional 2D sliceby-slice visualization and 3D volume rendering. For the 2D analysis, the artifact spread function (ASF) and Full Width at Half Maximum (FWHMM ${ }_{\text {ASF }}$ ) of the ASF were considered. The 3D quantitative analysis was based on the FWHM of disks profiles at $90^{\circ}$, noise and signal to noise ratio (SNR) at 0 - and 90 . A marked visual decrease of the artifact with reductions of $\mathrm{FWHM}_{\mathrm{ASF}}$ (2D) and $\mathrm{FWHM}_{90}$ (volume rendering) of $23.8 \%$ and $23.6 \%$, respectively, was observed. Although there was an expected increase in noise level, SNR values were preserved after deconvolution. Regardless of the methodology and visualization approach, the objective of reducing the out-of-plane artifact was accomplished. Both for the phantom and clinical case, the artifact reduction in the $z$ was markedly visible.
\end{abstract}

Index Terms-Blind deconvolution, breast tomosynthesis, data visualization, inverse problems, total variation minimization.

\section{INTRODUCTION}

$\mathrm{D}$ IGITAL Breast Tomosynthesis (DBT) has consolidated its position as an imaging technique used for breast cancer screening and diagnosis [1-5]. In DBT, a small number of lowdose projections acquired within a limited angular range are reconstructed to produce a three dimensional (3D) image of breast tissue. In the reconstruction, this angular limit restricts the spatial resolution in the direction perpendicular to the detector plane (z-direction), resulting in out-of-plane artifacts (blur in the z-direction). These artifacts are mostly produced by structures of high density that result in features of high attenuation and intensity (brighter) in DBT images. Examples are calcifications, biopsy needles and localization wires. The production of these artifacts could potentially obscure breast lesions and would limit the usability of DBT in interventional procedures. This is particularly important since DBT has a crucial role in guided biopsies for lesions such as architectural distortions (which can be occult in mammography and ultrasound images) $[6,7]$.

Currently, DBT images are analyzed with a two dimensional (2D) slice-by-slice visualization [8]. DBT presents, on average, sixty $2 \mathrm{D}$ slices per exam, which results in time-consuming analysis both in screening and daily clinical use [9-11]. Some studies have shown promising results in an attempt to reduce this time of analysis by highlighting some slices considered of interest and thus decreasing the number of slices to be analyzed $[12,13]$. However, these approaches result in an increase in false positives, requiring the radiologist to review multiple adjacent slices, sometimes leading to a more time consuming inspection [14]. Volume rendering is a $3 \mathrm{D}$ visualization approach which can display data from any angle, resulting in an immediate global inspection [15]. Therefore, DBT visualization by volume rendering may represent a complementary option in the analysis of DBT examinations [16] because it provides an understanding of the underlying data at once. In $2 \mathrm{D}$ visualization through $\mathrm{xy}$ planes along the $\mathrm{z}$-direction, the outof-plane artifact makes the bright structures visible in the infocus plane and replicated with lesser intensity to the underlying planes. In volume rendering this type of artifact remains quite evident with a noticeable blur at $90^{\circ}$ (visualization perpendicular to detector plate).

As the out-of-plane artifact is a current drawback in DBT imaging, there are several works addressing this topic. Most of them are focused on addressing this problem by filtering the projections [17-20], i.e. during reconstruction, with a few focusing on post-processing [21-23]. However, even with some of these techniques already implemented by the manufacturers [24], the artifact remains quite visible, with the characterization of calcifications and their morphology greatly affected by it [25].

The general imaging process can be denoted by the expression:

This work was supported by Universidade de Lisboa (PhD grant) and Fundação para a Ciência e Tecnologia - Portugal (Grant No. SFRH/BD/135733/2018 and FCT-IBEB Strategic Project UIDB/00645/2020).

${ }^{*}$ A. M. Mota, P. Almeida and N. Matela are with Instituto de Biofísica e Engenharia Biomédica, Faculdade de Ciências da Universidade de Lisboa, Lisboa, Portugal (e-mail: ammota@fc.ul.pt).

M. J. Clarkson is with Department of Medical Physics and Biomedical Engineering and the Centre for Medical Image Computing (CMIC) University College London, London, UK. 


$$
f=h \otimes u+n
$$

where $f$ and $u$ are the output (blurred) and the ideal data, respectively, $h$ represents the degradation function and $n$ is the noise model. In the spatial domain, $h$ is referred to as the point spread function (PSF), which is responsible for blurring $u$. Deconvolution is an inverse filtering process in which the effects of convolution by a PSF (blurring) should be inverted [26]. Given $f$ and some knowledge about $h$ (PSF) and $n$, the goal of deconvolution methods is to obtain an estimate of the original data, $u$ [27] using fast-Fourier transform algorithms [28].

One of the most difficult problems in image restoration is obtaining an adequate estimate of the PSF to use in deconvolution algorithms. The availability of suitable imaging equipment and phantoms which allow the correct determination of the PSF is limited [27]. When no information is available about the PSF, blind deconvolution can be used. The goal of blind deconvolution is to recover $u$ and the PSF from $f$ [28]. On the other hand, recovering the original data by inverse filtering involves noise amplification and DBT data are already noisy by nature due to the acquisition of low dose projections. Reducing noise without blurring or decreasing details and edge definition is a challenge in image processing. Total variation (TV) based minimization algorithms have achieved great success due to their ability to preserving edges while reducing image noise. $\mathrm{TV}$ is a quantity that characterizes how smoothly the intensity of an image is changing and it increases significantly in the presence of noise. Studies applying TV minimization to DBT data have grown significantly [29-35].

In this paper, a methodology to study the application of blind deconvolution to DBT data is proposed. Four different approaches to blind deconvolution are presented. Using the method described in Refs. [36] and [37] and noise regularization based on minimization of $\mathrm{TV}$ [33], a first estimate for the PSF is obtained. In addition, since the out-ofplane artifact is caused by features of high attenuation, representing sudden intensity variations, information about gradient magnitude is also taken into account during the deconvolution. The results are analyzed using real phantom data under 2D slice-by-slice visualization and 3D volume rendering with a compositing technique. The proposed methodology is also tested with one clinical DBT data set with a large calcification. To the best of our knowledge, this is the first study of out-of-plane artifact reduction through this type of deconvolution and noise regularization in DBT data.

\section{MATERIALS AND METHOdS}

\section{A. Acquisition, reconstruction and visualization}

To simulate high-density regions, a phantom built in our institution was used. A scheme and an image of the phantom are shown in Fig. 1. The phantom contains two columns of aluminum disks embedded in an acrylic background (Fig. 1 (b)). Considering the vertical direction, the aluminum disks were inserted in the central plaque of the phantom (dark gray in Fig. 1 (a)). The disks have different diameters and $1 \mathrm{~mm}$ thickness. For this study, the first column schematically represented in Fig. 1 (b) was considered (disks with diameters of $5.0 \mathrm{~mm}, 3.0 \mathrm{~mm}, 1.0 \mathrm{~mm}, 0.5 \mathrm{~mm}, 2.0 \mathrm{~mm}$ and $4.0 \mathrm{~mm}$, from top to bottom).

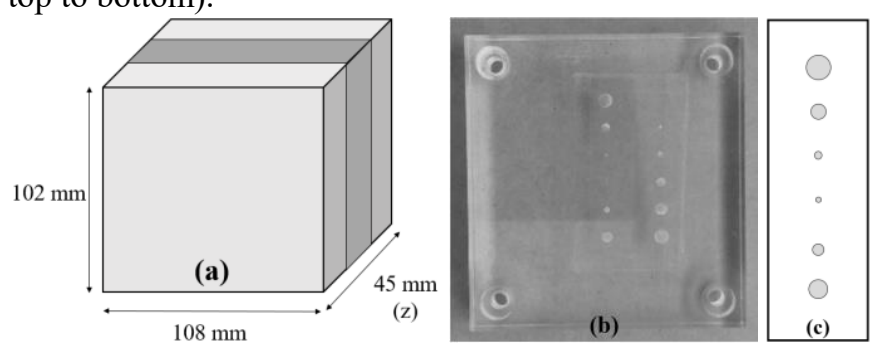

Fig. 1. (a) Scheme of the acrylic phantom used. (b) Image of the phantom simulating breast tissue and lesions of high attenuation (aluminum disks of different diameters and $1 \mathrm{~mm}$ thickness). (b) Scheme of the disks in the first column (top to bottom): $5.0 \mathrm{~mm}, 3.0 \mathrm{~mm}, 1.0 \mathrm{~mm}$, $0.5 \mathrm{~mm}, 2.0 \mathrm{~mm}$ and $4.0 \mathrm{~mm}$, respectively.

Additionally, one clinical DBT data set with a large calcification of an anonymous patient was selected from a clinical facility database (Hospital da Luz S.A., Lisbon, Portugal). Both phantom and clinical data set were acquired with a Siemens MAMMOMAT Inspiration system (Siemens AG, Healthcare Sector, Erlangen, Germany). The reconstructions have voxel sizes of $0.085 \times 0.085 \times 1 \mathrm{~mm}^{3}$ and were obtained with the manufacturer algorithm which uses Filtered Back Projection with some post-processing to reduce artifacts and image blurring [38].

The $3 \mathrm{D}$ visualization software was developed in $\mathrm{C}++$ using the Visualization Toolkit library (VTK) version 7.1.0 [39, 40]. The methodologies in study were analyzed with $3 \mathrm{D}$ volume rendering visualization using ray casting and compositing. Different rendering parameters yield different images. In this way, the parameters were fixed for all situations so that a correct comparison could be made.

\section{B. PSF estimation in z-direction}

The phantom was reconstructed in the xy plane and z-depth. As the out-of-plane artifact is present in the z-direction, its study was performed considering a PSF in the xz plane. By combining the methods described in Refs. [36] and [37], one xz plane containing the entire $0.5 \mathrm{~mm}$ disk distribution was chosen. In that plane, a region of interest (ROI) containing the $0.5 \mathrm{~mm}$ disk and some background (BG) was extracted. To reduce the noise variation, a TV minimization filter was applied to this region [33]. In order to achieve the minimum value of $\mathrm{TV}$, several values of Lagrange parameter were applied to the longitudinal direction. From this fine analysis, Lagrange parameter $=156$ has shown to be the one that allows the maximum reduction of TV and, for this reason, it was the chosen value for the application of the filter in this direction. In addition, the mean intensity of two BG ROIs was calculated and subtracted from that region. This region was thus considered as the first estimation of the PSF in z-direction.

\section{Blind deconvolution}

With blind deconvolution, an approximation of the true data $u$ (deblurred) and the PSF can be recovered using observed data $f$ (blurred) and an initial estimation of the PSF. As described in Fig. 2, four approaches using blind deconvolution were studied 


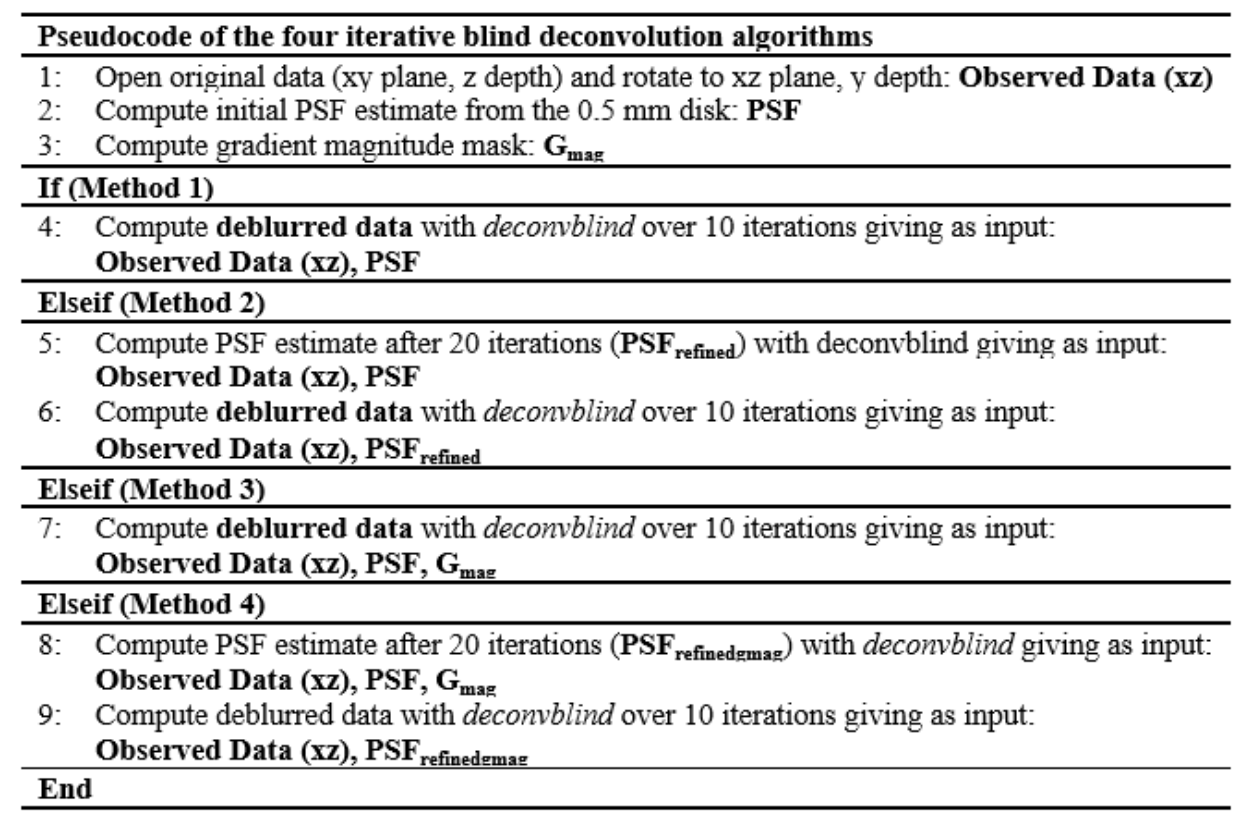

Fig. 2. Pseudocode of the four methods implemented in order to study the application of blind deconvolution in the reduction of out-of-plane artifacts in DBT data.

in this work.

Method 1 is the simplest one. An initial estimation of the PSF, obtained as described in section II.B., and rotated data (so that the deconvolution occurs in xz planes) were given as input to blind deconvolution. After a certain number of iterations, the deblurred data were obtained. The second method is similar to the first one, except that prior to data deblurring, the deconvolution algorithm ran for 20 iterations and the resulting PSF at the end of these iterations was given as input, together with the blurred data, for the deconvolution of the data itself. The choice of using a PSF after 20 iterations has relied on empirical studies made during the experiment.

Methods 3 and 4 are identical to methods 1 and 2, respectively, with the exception that as input, in addition to the PSF estimation and blurred data, a mask corresponding to the gradient magnitude of the same data was also considered. The gradient magnitude allows to detect sudden transitions of intensity corresponding to the high attenuation features which originate the artifacts. The use of this mask determines how much the voxel at the corresponding position in the input data is considered. In this way, these sudden changes were intended to have zero weight so that their influence on the deconvolution was minimal and to reduce the contrast-related image artifacts. After the gradient calculation, its complement was obtained and a binarization was carried out, where values of zero correspond to that transitions.

Using observed data as the reference, structural similarity index (SSIM) values calculated for deblurred data at the end of each iteration were considered for stopping the iterative process. In order to preserve the fidelity of the results down to the smallest structure, SSIM was calculated over an area including the smallest disk $(0.5 \mathrm{~mm})$, considering all slices. The deconvolution algorithms were stopped when the SSIM dropped below 0.7 .

The algorithms were implemented based on the MATLAB R2016b function deconvblind [41] and an Intel ${ }^{\circledR}$ Core ${ }^{\text {TM }}$ i5-
5200U CPU (2.20 GHz)@8 GB of memory computer was used.

\section{Data analysis}

The artifact in the z-direction was quantitatively analyzed through standard 2D slice-by-slice visualization and also through $3 \mathrm{D}$ volume rendering. For the $2 \mathrm{D}$ analysis, the artifact spread function (ASF) in the z-direction was calculated for each disk as in (2) in order to verify the consistency of the results at contrast and noise level.

$$
\operatorname{ASF}(z)=\left(\frac{\mu_{D}(z)-\mu_{B G}(z)}{\sigma_{B G}(z)}\right) /\left(\frac{\mu_{D}\left(z_{0}\right)-\mu_{B G}\left(z_{0}\right)}{\sigma_{B G}\left(z_{0}\right)}\right)
$$

In (2), $z_{0}$ is the slice location of the in-focus plane of the disk and $\mathrm{z}$ an off-focus plane. $\mu_{\mathrm{D}}$ and $\mu_{\mathrm{BG}}$ stand for mean pixel values in the ROI over the disk and BG, respectively, $\sigma_{\mathrm{BG}}$ stands for standard deviation in BG ROI. The values of $\mu_{\mathrm{BG}}$ and $\sigma_{\mathrm{BG}}$ were obtained using two circular ROIs arranged on either side of the disk. The ASF curve is related with the extent of the artifact, namely its width. In this way, the full width at half maximum $\left(\mathrm{FWHM}_{\mathrm{ASF}}\right)$ of a Gaussian curve fitted to the ASF of each disk, for each method, was calculated. To evaluate the accuracy between ASF points and the Gaussian curve, the coefficient of determination $\left(\mathrm{R}^{2}\right)$ was obtained for each fitting.

In volume rendering, when changing a camera's azimuth, its position rotates around the focal point. The result is a horizontal rotation (to the left or right) of the camera keeping the distance to the focal point constant [40]. In this case, two different angles were used to visualize the DBT data: $0^{\circ}$, which corresponds to the visualization parallel to the detector (i.e. projection is made on the xy planes along $\mathrm{z}$ ); and $90^{\circ}$, which is the visualization perpendicular to the detector plate (i.e. projection is made on the $x z$ planes along $y$ ). For the 3D quantitative evaluation using volume rendering, three figures of merit were obtained for each disk: FWHM (at $90^{\circ}$ ), noise (at $0^{\circ}$ and $90^{\circ}$ ) and signal to noise 
ratio (SNR) (at $0^{\circ}$ and $90^{\circ}$ ). The profile of each disk at $90^{\circ}$ was obtained and the $\mathrm{FWHM}_{90^{\circ}}$ of a Gaussian curve fitted to each profile was considered as an indicator of the spreading size of the artifact. In this case, the $\mathrm{R}^{2}$ values was also obtained for each fitting. Noise was calculated as in (3) and, in order to study the relation between signal strength and BG noise, SNR was calculated using (4).

$$
\begin{aligned}
& \text { Noise }=\frac{\mu_{B G}}{\sigma_{B G}} \\
& \text { SNR }=\frac{\mu_{D}}{\sigma_{B G}}
\end{aligned}
$$

For qualitative analysis, displays of the phantom and one clinical case obtained with volume rendering visualization at $0^{\circ}$ and $90^{\circ}$ are presented.

\section{REsults}

The SSIM for deblurred data using observed data as reference remained above 0.7 until iteration number four (Fig. 3). The SSIM values obtained for this iteration were $0.75,0.71,0.81$ and 0.79 for method 1, 2, 3 and 4 , respectively. In this way, the stop iteration was the fourth, achieved approximately after 3.7 minutes for the four methods.

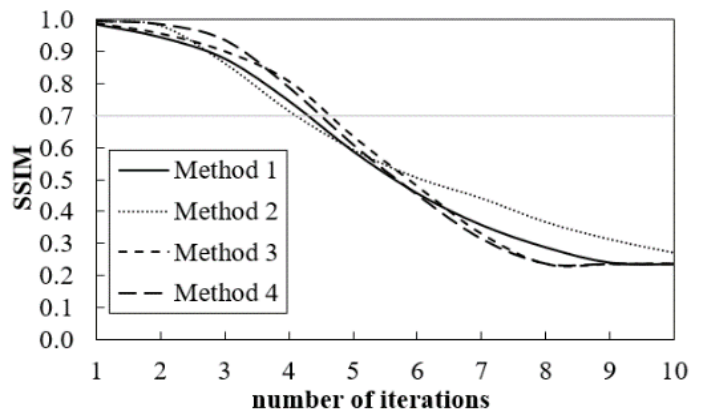

Fig. 3. SSIM values obtained for deblurred data with each iterative blind deconvolution method, as a function of iteration number.

\section{A. 2D analysis}

The obtained ASF for deblurred data with each method and original data are presented from Fig. 5 (a) to Fig. 5 (f), for the six
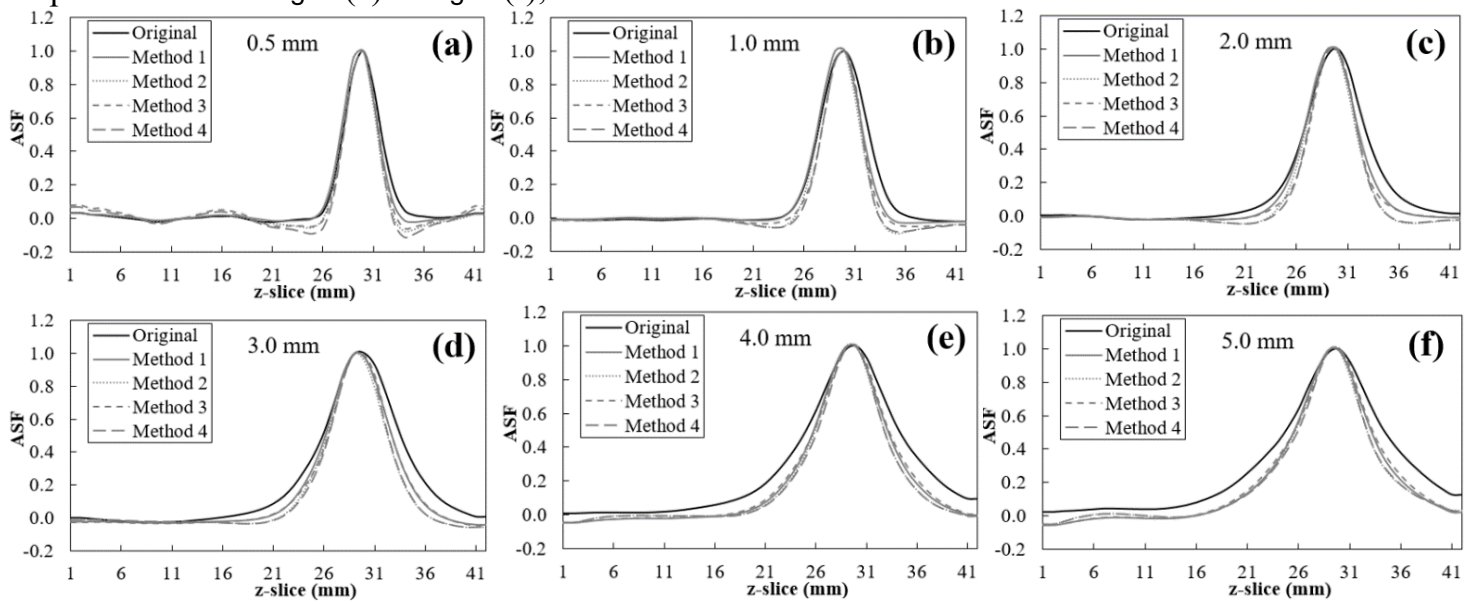
disk. disk. 


\section{B. 3D analysis}

The $\mathrm{FWHM}_{90^{\circ}}$ values obtained for the profile of each disk at $90^{\circ}$ are presented in Fig. 6. Gaussian curve fittings for each profile showed $\mathrm{R}^{2}$ values above 0.91 .

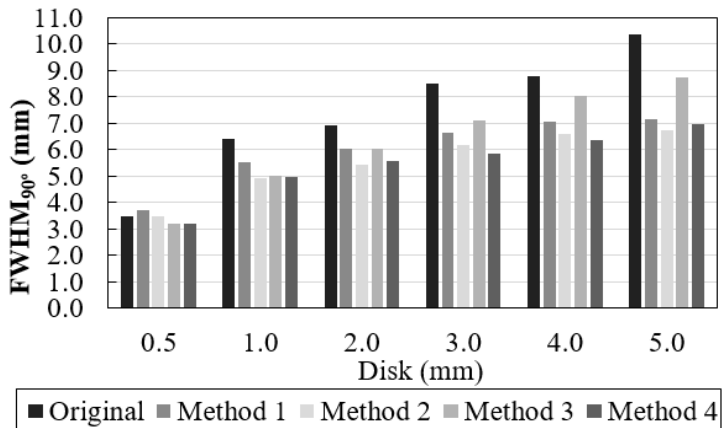

Fig. 6. FWHM9o values of a Gaussian curve fitted to the profile of each disk at $90^{\circ}(0.5 \mathrm{~mm}, 1.0 \mathrm{~mm}, 2.0 \mathrm{~mm}, 3.0 \mathrm{~mm}, 4.0 \mathrm{~mm}$ and $5.0 \mathrm{~mm})$, for each method and original data.

As for the $\mathrm{FWHM}_{\mathrm{ASF}}$ values, the percentage of variation between $\mathrm{FWHM}_{90^{\circ}}$ values of blurred and deblurred data with each method, are shown in Table 2.

TABLE 2

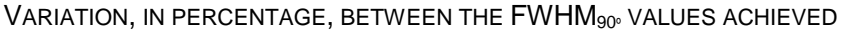
FOR THE ORIGINAL DATA AND AFTER DECONVOLUTION WITH EACH METHOD.

TAKING INTO ACCOUNT THE DIFFERENT DISKS, THE AVERAGE OF THE OBTAINED VARIATIONS WITH EACH METHOD IS PRESENTED IN THE LAST ROW.

\begin{tabular}{ccccc}
\hline \multicolumn{5}{c}{$\Delta_{\text {FWHM }}$ 90 $^{\circ}(\%)$} \\
\hline Disk $(\mathrm{mm})$ & Method 1 & Method 2 & Method 3 & Method 4 \\
\hline 0.5 & 7.4 & 0.8 & -7.4 & -8.0 \\
1.0 & -13.5 & -23.5 & -22.1 & -22.4 \\
2.0 & -12.9 & -21.8 & -12.5 & -19.4 \\
3.0 & -21.5 & -27.1 & -16.1 & -31.1 \\
4.0 & -19.7 & -25.1 & -8.4 & -27.6 \\
5.0 & -30.9 & -35.1 & -15.6 & -33.0 \\
\hline mean & -15.2 & -21.9 & -13.7 & -23.6 \\
\hline
\end{tabular}

Results of noise and SNR at $0^{\circ}$ and $90^{\circ}$ obtained in the phantom original and deblurred images for each disk are presented in Fig. 7 and Fig. 8, respectively. For a qualitative inspection, volume rendering images of the phantom at $0^{\circ}$ and $90^{\circ}$ are presented in

Fig. 9 and

Fig. 10, respectively.

To evaluate the consistency of the proposed methodology, method 4 was applied to one clinical data set and it was stopped at iteration number four. For clinical data, the method 4 took approximately 2 minutes to complete the four iterations. 2D displays of composite volume rendering obtained at $0^{\circ}$ and $90^{\circ}$ are shown in Fig. 11.

\section{DISCUSSION}

Four methods using TV minimization and blind deconvolution to reduce out-of-plane artifacts in DBT imaging have been presented. The artifact in the z-direction was quantitatively analyzed through standard 2D slice-by-slice visualization and with $3 \mathrm{D}$ volume rendering. The $2 \mathrm{D}$ evaluation was focused on the ASF curves of each disk and respective

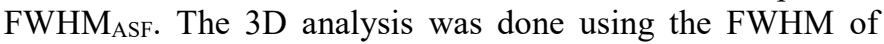
disks profiles at $90^{\circ}\left(\mathrm{FWHM}_{90^{\circ}}\right)$, noise and SNR at $0^{\circ}$ and $90^{\circ}$. $2 \mathrm{D}$ displays of volume rendering at $0^{\circ}$ and $90^{\circ}$ were considered for visual inspection.

In phantom data we observed that, regardless of the methodology and visualization approach, the objective of reducing the out-of-plane artifact was accomplished for all disk sizes (Table 1 and Table 2). In 2D, ASF was narrowed by all methods, for all disks, near the in-focus slice (slice number 30 ). Although all disks have a thickness of $1 \mathrm{~mm}$ (in z), it was clear that their diameter (in xy) affects the size of the artifact. For example, if we compare ASF of $1.0 \mathrm{~mm}$ disk (Fig. 5 (b)) with ASF of $5.0 \mathrm{~mm}$ disk (Fig. 5 (f)), it is clearly evident that for the larger diameter in $\mathrm{xy}$, the artifact in the z-direction is larger.
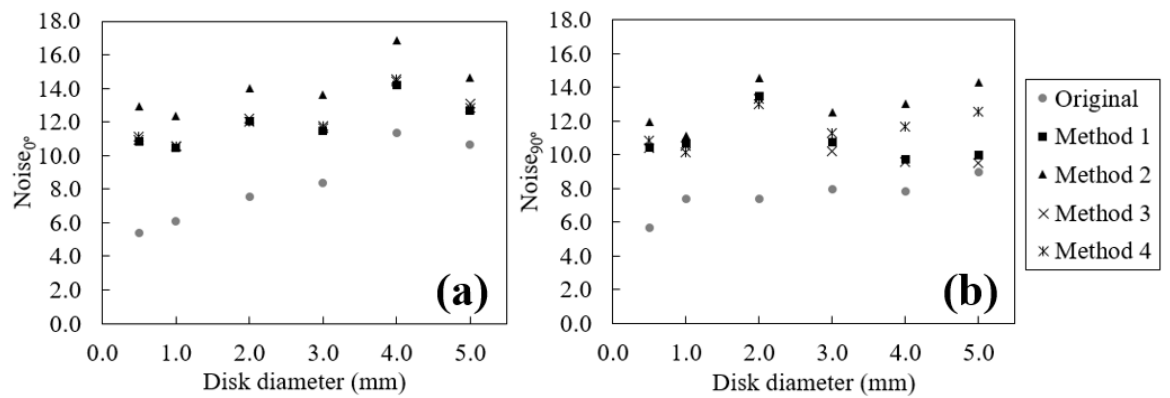

Fig. 7. Noise at $0^{\circ}$ (a) and $90^{\circ}$ (b) obtained for phantom original and deblurred data with each method plotted as a function of disk diameter.
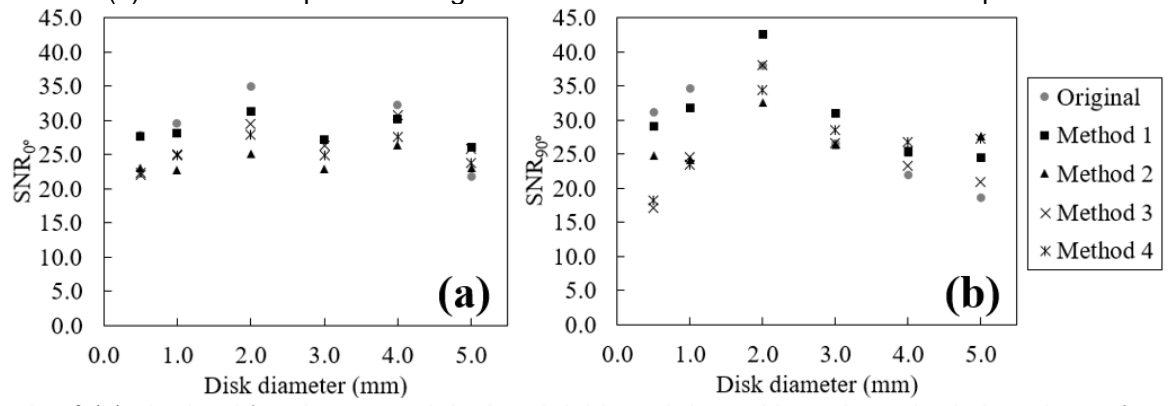

Fig. 8. SNR at $0^{\circ}(\mathrm{a})$ and $90^{\circ}$ (b) obtained for phantom original and deblurred data with each method plotted as a function of disk diameter. 


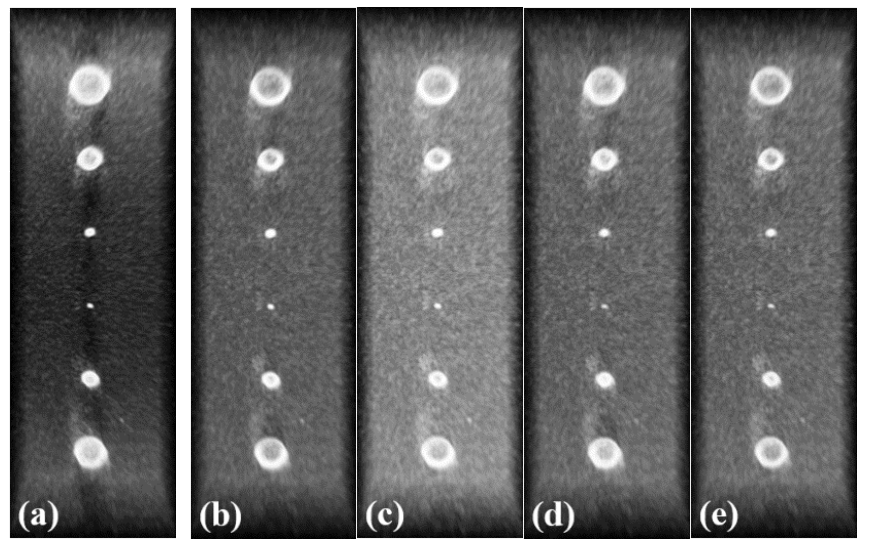

Fig. 9. 2D displays of composite volume rendering visualization obtained at $0^{\circ}$ for blurred (a) and deblurred data with method 1 (b), method 2 (c), method 3 (d) and method 4 (e).
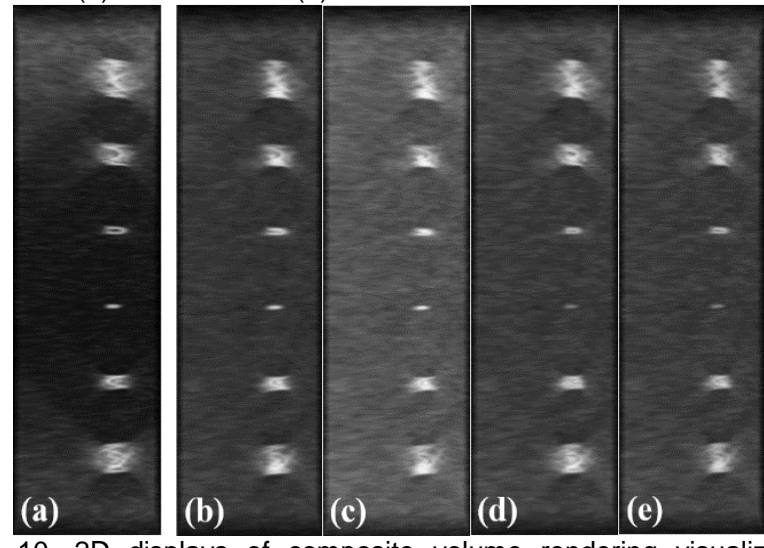

Fig. 10. 2D displays of composite volume rendering visualization obtained at $90^{\circ}$ for blurred (a) and deblurred data with method 1 (b), method 2 (c), method 3 (d) and method 4 (e).
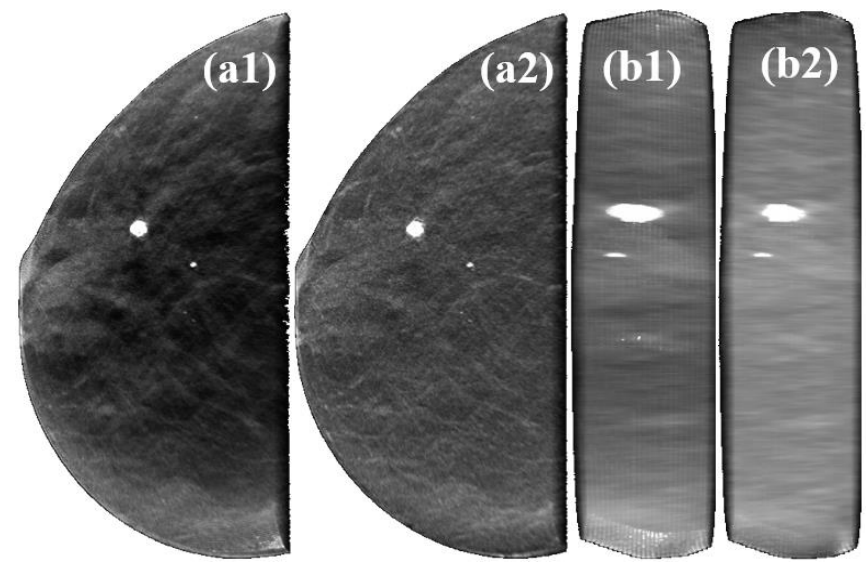

Fig. 11. 2D displays of composite volume rendering visualization obtained at 0 and 90 degrees ( $a$ and b, respectively) for blurred ( $a 1$ and b1) and deblurred data with method 4 (a2 and b2).

These results were confirmed by FWHM ASF $_{\text {values in Fig. } 4 .}$ The $5.0 \mathrm{~mm}$ disk presented the highest values of $\mathrm{FWHM}_{\mathrm{ASF}}$ for all cases (original data and deblurred data) and the $0.5 \mathrm{~mm}$ disk the lowest $\mathrm{FWHM}_{\mathrm{ASF}}$ values. Method 4 yielded the lowest $\mathrm{FWHM}_{\mathrm{ASF}}$ values for all disks, resulting in the method that, on average, achieves a larger reduction on the ASF width: $23.8 \%$ (Table 1). With method 1 and 3 , average reductions in FWHM $_{\mathrm{ASF}}$ of $14.0 \%$ and $17.7 \%$ were obtained, respectively, whereas method 2 achieved $21.3 \%$. The $\mathrm{R}^{2}$ values obtained after fitting a Gaussian curve to the ASF points were higher than 0.95 , supporting the validity of the approximations. In this way, the FWHM $_{\text {ASF }}$ values were considered as an estimate of the ASF curve width.

With volume rendering visualization, realistic computergenerated images of a 3D scene are obtained, yielding a true depth perception available through angular rotation. In this particular study, since the out-of-plane artifact propagates through multiple slices, we were interested in what happens in the z-direction. For that reason, a rotation of $90^{\circ}$ was done and some quantitative analysis was performed on these rendered images. Through Fig. 4 and Fig. 6 we can see that the results from both visualizations followed the same pattern. Disks with largest dimensions at $0^{\circ}$ (xy planes) have a higher FWHM value at $90^{\circ}$ and also a higher deblurring after deconvolution methods. In Table 2, the $5.0 \mathrm{~mm}$ disk presented reductions in the spreading size of the artifact in the order of $30 \%$, whereas 1.0 $\mathrm{mm}$ disk was at $20 \%$. The mean values of the variations in Table 1 (last row) are comparable to those in Table 2. Taking all disks into account, the mean reductions of $\mathrm{FWHM}_{90^{\circ}}$ values in volume rendering were $15.2 \%, 21.9 \%, 13.7 \%$ and $23.6 \%$ for method 1,2, 3 and 4, respectively. The $\mathrm{R}^{2}$ values obtained after fitting a Gaussian curve to the volume rendering profiles at $90^{\circ}$ were higher than 0.90 , showing once again a good adjustment. Additionally, at $90^{\circ}$ (Fig. 10), there was a noticeable disk blur reduction in all deblurred images. This is in line with the numerical reduction of $\mathrm{FWHM}_{\mathrm{ASF}}$ and $\mathrm{FWHM}_{90^{\circ}}$ values. It is important to notice that, neither in the ASF nor in rendered images, were observed ringing artifacts often resultant from this type of deconvolution due to the limited bandwidth of the system. However, for the smaller disk, some overshoot values were observed in the ASF of methods 3 and 4 (although there are no negative effects in the quality of the images resulting from these methods). This can be explained by the location of the ROIs and by the fact that the ROI covering the $0.5 \mathrm{~mm}$ disk is the smallest one (being more sensitive to small variations). Additionally, PSF-based methods are known to cause edge artifacts which appear as an overshoot at the sharp transitions of the intensity of the phantom. To address this issue, an improvement in the PSF estimation should be considered.

Deconvolution methods introduced noise at $0^{\circ}$ and $90^{\circ}$ (Fig. 7). The noise for each disk was measured considering a $B G$ region surrounding the disk. In this way, the differences observed are essentially due to the spatial dependence of noise on DBT and not to the disk in question. Despite increasing it, deconvolution methods homogenized data noise level, with smaller differences obtained in the different spatial regions. These results are perceptible in Fig. 9 and Fig. 10. In original rendered images (Fig. 9 (a) and Fig. 10 (a)), it is visible that top and bottom phantom regions, which coincide with larger disks $(5.0 \mathrm{~mm}$ and $4.0 \mathrm{~mm}$, respectively), present a greater noise level than the center region (near the $0.5 \mathrm{~mm}$ disk). In phantom rendered images resultant from deconvolution methods (Fig. 9 (b)-(e) and Fig. 10 (b)-(e)), this difference between top/bottom regions and the center is not noticeable. On the other hand, SNR values of each disk at $0^{\circ}$ were very close in the original and deblurred images (Fig. 8 (a)). This means that although there has been an increase in noise level, the increase in signal strength on each disk has allowed a balance. SNR was 
preserved and the visualization of each disk was not affected. At $90^{\circ}$ (Fig. 8 (b)), a decrease in SNR values for the smaller disks $(0.5 \mathrm{~mm}$ and $1.0 \mathrm{~mm})$ was observed for all methods whereas, for the remaining disks, SNR values increased after deconvolution. By comparing the original (Fig. 10 (a)) and deblurred phantom images (Fig. 10 (b)-(e)), this decrease in SNR is expressed with a loss of contrast in smaller disks, in particular with methods 3 and 4 . However, the diameter of the disks and their shapes after deconvolution remain intact, preserving the structural information correctly. In any case, this fact should be carefully analyzed in the future.

From numerical results of Table 1 and Table 2, we observed that giving as input a PSF which was already estimated after 20 iterations improves the results (method 2 and 4). This happens because the initial estimate of PSF (after 20 iterations) is closer to the true PSF, improving the deconvolution algorithm results for the same number of iterations. The difference between method 2 and 4 was the introduction of the gradient magnitude mask. Due to the high intensity of the disks, the deconvolution process can result in noise and blurring, essentially on smaller disks (such as $0.5 \mathrm{~mm}$ disk), because those are more susceptible to minor fluctuations. Results obtained with method 2 were an example of that. Considering Table 2, method 2 presented an equal or superior performance than method 4 on the largest disks (for example, $5.0 \mathrm{~mm}$ and $2.0 \mathrm{~mm}$ ), whereas on a smaller disk $(0.5 \mathrm{~mm})$ the $\mathrm{FWHM}_{90^{\circ}}$ even increased compared to the original data. The methods which used the information of the gradient magnitude mask, excluding the voxels around the disks during the restoration (assigning zero weight to them) were able to preserve this information, without blurring it. This resulted in improvements of $7.4 \%(\operatorname{method} 3)$ and $8.0 \%(\operatorname{method} 4)$ in the deblurring of the smaller disk.

The method which achieved the maximum mean reduction of $\mathrm{FWHM}_{\mathrm{ASF}}$ and $\mathrm{FWHM}_{90^{\circ}}(\operatorname{method} 4)$ was applied to the clinical data. The stop iteration according to the criterion of $\mathrm{SSIM} \geq 0.7$ was the fifth. However, to keep the comparison with phantom data reliable, the clinical images presented were obtained in iteration number four $($ SSIM $=0.87)$. The results obtained with the clinical data (Fig. 11) were in accordance with the phantom results. There was a visible reduction of the big calcification blur at $90^{\circ}$, with an increase of noise level at $0^{\circ}$. The preliminary clinical data results here presented are intended to consolidate the results obtained with the phantom. Additional studies with volume rendering should be considered in the future. For example, the size of the calcification should be known in order to perform a quantitative study and thus to draw conclusions in this regard.

Although beam hardening artifact is extremely rare in DBT [42], it was observed in the images of the phantom at $0^{\circ}$ (Fig. 9), namely in the larger disks. This effect is present in the original image and remains evident in the images after deconvolution. In this way, it was not introduced by blind deconvolution itself. The phantom disks under study have a much higher density than the acrylic background, producing a very abrupt transition, thus resulting in this effect on the images. However, the images that radiologists analyze on daily basis are clinical images, similar to those in Fig. 11, where this artifact was not observed, neither in the original data nor after the application of the blind deconvolution method.

For future work, we plan to improve the PSF estimation and test other deconvolution approaches, such as Wiener and LucyRichardson. Deconvolution should also be optimized for the noise level in data (not only at the moment of PSF estimation). In addition, as the presented methods may produce different gains and results with other scanners (with different angular range and number of projections), the study should be extended to different acquisition scenarios.

\section{CONCLUSION}

In DBT data acquisition there are two major aspects that greatly influence the reconstructed images: the low dose projections and the angular limit. These factors produce noisy images and out-of-plane artifacts where high intensity structures lay in the in-focus plane but also spread along other planes (which may lead to a significant decrease in DBT image quality in the areas surrounding a lesion of interest). As the outof-plane artifact is a current drawback in DBT imaging, four methods based on TV regularization and blind deconvolution have been studied. The obtained results were analyzed through standard 2D slice-by-slice visualization and through 3D volume rendering.

The methodologies were tested with phantom and clinical DBT data. Both quantitative and qualitative analysis performed showed the relevance of this approach in improving the image quality in DBT by reducing the out-of-plane artifact without introducing other artifacts, typical of the deconvolution in noisy data. This methodology can be very useful for future tools in DBT, such as computer assisted diagnosis and DBT-guided needle biopsies.

This study presents the particularity of visual analysis being performed through a truly $3 \mathrm{D}$ visualization (volume rendering). In this way, it is possible to get a sense of the impact of these algorithms on the data in a direct way, by visualizing the DBT data at once from several angles (in this case, from $0^{\circ}$ and $90^{\circ}$ ).

\section{REFERENCES}

[1] G. Gennaro, A. Toledano, C. di Maggio, E. Baldan, E. Bezzon, M. La Grassa, L. Pescarini, I. Polico, A. Proietti, A. Toffoli, and P. C. Muzzio, "Digital breast tomosynthesis versus digital mammography: a clinical performance study," Eur Radiol, vol. 20, pp. 1545-1553, Jul. 2010.

[2] K. R. Brandt, D. A. Craig, T. L. Hoskins, T. L. Henrichsen, E. C. Bendel, S. R. Brandt, and J. Mandrekar, "Can Digital Breast Tomosynthesis Replace Conventional Diagnostic Mammography Views for Screening Recalls Without Calcifications? A Comparison Study in a Simulated Clinical Setting," American Journal of Roentgenology, vol. 200, pp. 291-298, Feb. 2013.

[3] M. M. Bonafede, V. B. Kalra, J. D. Miller, and L. L. Fajardo, "Value analysis of digital breast tomosynthesis for breast cancer screening in a commercially-insured US population," ClinicoEconomics and Outcomes Research: CEOR, vol. 7, pp. 53-63, 2015.

[4] Y. Gao, J. S. Babb, H. K. Toth, L. Moy, and S. L. Heller, "Digital Breast Tomosynthesis Practice Patterns Following 2011 FDA Approval: A Survey of Breast Imaging Radiologists," Acad Radiol, vol. 24, pp. 947953, Aug. 2017.

[5] S. Destounis, A. Santacroce, and A. Arieno, "DBT as a Screening Tool and a Diagnostic Tool," Current Breast Cancer Reports, vol. 9, pp. 264271, Dec. 2017.

[6] L. Partyka, A. P. Lourenco, and M. B. Mainiero, "Detection of Mammographically Occult Architectural Distortion on Digital Breast Tomosynthesis Screening: Initial Clinical Experience," American Journal of Roentgenology, vol. 203, pp. 216-222, Jul. 2014.

[7] M. A. Durand, S. Wang, R. J. Hooley, M. Raghu, and L. E. Philpotts, "Tomosynthesis-detected Architectural Distortion: Management 
Algorithm with Radiologic-Pathologic Correlation," Radiographics, vol. 36, pp. 311-321, 2016.

[8] I. Sechopoulos, "A review of breast tomosynthesis. Part I. The image acquisition process," Med Phys, vol. 40, p. 014301, Jan. 2013.

[9] W. F. Good, G. S. Abrams, V. J. Catullo, D. M. Chough, M. A. Ganott, C. M. Hakim, and D. Gur, "Digital breast tomosynthesis: a pilot observer study," AJR Am J Roentgenol, vol. 190, pp. 865-9, Apr. 2008.

[10] D. Gur, G. S. Abrams, D. M. Chough, M. A. Ganott, C. M. Hakim, R. L. Perrin, G. Y. Rathfon, J. H. Sumkin, M. L. Zuley, and A. I. Bandos, "Digital breast tomosynthesis: observer performance study," AJR Am J Roentgenol, vol. 193, pp. 586-91, Aug. 2009.

[11] F. Caumo, M. Zorzi, S. Brunelli, G. Romanucci, R. Rella, L. Cugola, P. Bricolo, C. Fedato, S. Montemezzi, and N. Houssami, "Digital Breast Tomosynthesis with Synthesized Two-Dimensional Images versus Full-Field Digital Mammography for Population Screening: Outcomes from the Verona Screening Program," Radiology, vol. 287, pp. 37-46, 2018.

[12] R. A. Benedikt, J. E. Boatsman, C. A. Swann, A. D. Kirkpatrick, and A. Y. Toledano, "Concurrent Computer-Aided Detection Improves Reading Time of Digital Breast Tomosynthesis and Maintains Interpretation Performance in a Multireader Multicase Study," American Journal of Roentgenology, vol. 210, pp. 685-694, Mar. 2017.

[13] C. Balleyguier, J. Arfi-Rouche, L. Levy, P. R. Toubiana, F. CohenScali, A. Y. Toledano, and B. Boyer, "Improving digital breast tomosynthesis reading time: A pilot multi-reader, multi-case study using concurrent Computer-Aided Detection (CAD)," Eur J Radiol, vol. 97 , pp. 83-89, Dec. 2017.

[14] P. M. Tchou, T. M. Haygood, E. N. Atkinson, T. W. Stephens, P. L. Davis, E. M. Arribas, W. R. Geiser, and G. J. Whitman, "Interpretation Time of Computer-aided Detection at Screening Mammography," Radiology, vol. 257, pp. 40-46, 2010.

[15] P. Suetens, "Medical image analysis," in Fundamentals of Medical Imaging, 2nd ed New York: Cambridge University Press, 2009, pp. 159-189.

[16] J. E. Venson, J. C. Albiero Berni, C. Edmilson da Silva Maia, A. M. Marques da Silva, M. Cordeiro d'Ornellas, and A. Maciel, "A CaseBased Study with Radiologists Performing Diagnosis Tasks in Virtual Reality," Stud Health Technol Inform., vol. 245, pp. 244-248., 2017.

[17] T. Wu, R. H. Moore, and D. B. Kopans, "Voting strategy for artifact reduction in digital breast tomosynthesis," Med Phys, vol. 33, pp. 24612471, 2006.

[18] S. Abdurahman, A. Jerebko, T. Mertelmeier, T. Lasser, and N. Navab, "Out-of-Plane Artifact Reduction in Tomosynthesis Based on Regression Modeling and Outlier Detection," Berlin, Heidelberg, 2012, pp. 729-736.

[19] J. Wicklein, A. Jerebko, L. Ritschl, and T. Mertelmeier, "Metal and calcification artifact reduction for digital breast tomosynthesis," in SPIE Medical Imaging, 2017, p. 7.

[20] M. Dustler, J. Wicklein, H. Förnvik, J. Boita, P. Bakic, and K. Lång, "High-attenuation artifact reduction in breast tomosynthesis using a novel reconstruction algorithm," Eur J Radiol, vol. 116, pp. 21-26, Jul. 2019.

[21] Z. Kolitsi, G. Panayiotakis, and N. Pallikarakis, "A method for selective removal of out-of-plane structures in digital tomosynthesis," Med Phys., vol. 20, pp. 47-50. doi: 10.1118/1.597060., 1993.

[22] X. Sun, W. Land, and R. Samala, "Deblurring of tomosynthesis images using 3D anisotropic diffusion filtering," in Medical Imaging, 2007, p. 11.

[23] K. Bliznakova, Z. Bliznakov, and N. Pallikarakis, "An improved algorithm for out-of-plane artifacts removal in digital tomosynthesis reconstructions," Berlin, Heidelberg, 2010, pp. 367-370.

[24] A. Jerebko, "Out of plane artifact reduction in digital breast tomosynthesis and ct," 2016.

[25] S. Mall, S. Lewis, P. Brennan, J. Noakes, and C. Mello-Thoms, "The role of digital breast tomosynthesis in the breast assessment clinic: a review," Journal of Medical Radiation Sciences, vol. 64, pp. 203-211, 2017

[26] B. Jähne, Digital Image Processing: Concepts, Algorithms, and Scientific Applications, 3rd ed. Berlin, Germany: Springer, 1995.

[27] R. C. Gonzalez and R. E. Woods, Digital Image Processing Using MATLAB, 3rd ed. Upper Saddle River, New Jersey: Pearson Prentice Hall, 2008.

[28] G. R. Ayers and J. C. Dainty, "Iterative blind deconvolution method and its applications," Optics Letters, vol. 13, pp. 547-549, Jul. 1988.
[29] E. Y. Sidky, X. Pan, I. S. Reiser, R. M. Nishikawa, R. H. Moore, and D. B. Kopans, "Enhanced imaging of microcalcifications in digital breast tomosynthesis through improved image-reconstruction algorithms," Med Phys, vol. 36, pp. 4920-32, Nov. 2009.

[30] S. Seyyedi, K. Cengiz, M. Kamasak, and I. Yildirim, "An objectoriented simulator for 3D digital breast tomosynthesis imaging system," Comput Math Methods Med, vol. 2013, pp. 250689-250689, 2013.

[31] K. Michielsen, J. Nuyts, L. Cockmartin, N. Marshall, and H. Bosmans, "Design of a model observer to evaluate calcification detectability in breast tomosynthesis and application to smoothing prior optimization," Med Phys, vol. 43, pp. 6577-6587, 2016.

[32] J. Krammer, S. Zolotarev, I. Hillman, K. Karalis, D. Stsepankou, V. Vengrinovich, J. Hesser, and T. M. Svahn, "Evaluation of a new image reconstruction method for digital breast tomosynthesis: effects on the visibility of breast lesions and breast density," The British Journal of Radiology, vol. 92, 2019.

[33] A. M. Mota, N. Matela, N. Oliveira, and P. Almeida, "Total variation minimization filter for DBT imaging," Med Phys, vol. 42, pp. 28272836,2015

[34] A. M. Mota, N. Matela, N. Oliveira, and P. Almeida, "An iterative algorithm for Total Variation minimization in DBT imaging," in VipIMAGE 2015, , Tenerife, Spain, 2015, pp. 119-122.

[35] A. M. Mota, N. Oliveira, P. Almeida, and N. Matela, "3D Total Variation Minimization Filter for Breast Tomosynthesis Imaging," Malmo, Sweden, 2016, pp. 501-509.

[36] Y.-H. Hu, B. Zhao, and W. Zhao, "Image artifacts in digital breast tomosynthesis: Investigation of the effects of system geometry and reconstruction parameters using a linear system approach," Med Phys, vol. 35, pp. 5242-5252, 2008.

[37] S. Richard and E. Samei, "3D task-based performance assessment metrics for optimization of performance and dose in breast tomosynthesis," in SPIE Medical Imaging, 2011, p. 6.

[38] Siemens. (2015, February). MAMMOMAT Inspiration - Tomosynthesis Option. Available: https://www.accessdata.fda.gov/cdrh_docs/pdf14/P140011c.pdf

[39] VTK. (2020, February). Visualization Toolkit - VTK. Available: http://www.vtk.org/

[40] W. Schroeder, K. Martin, and B. Lorensen, The Visualization Toolkit: An Object-oriented Approach to 3D Graphics, 4rd ed. USA: Kitware, 2006.

[41] MathWorks. (2020, January). MATLAB deconvblind function. Available:

https://www.mathworks.com/help/images/ref/deconvblind.html

[42] W. R. Geiser, S. A. Einstein, and W.-T. Yang, "Artifacts in Digital Breast Tomosynthesis," American Journal of Roentgenology, vol. 211, pp. 926-932, 2018/10/01 2018. 